BNL- 52546

\title{
THEORY OF TAPERED LASER COOLING
}

\author{
HIROMI OKAMOTO \\ and \\ JIE WEI
}

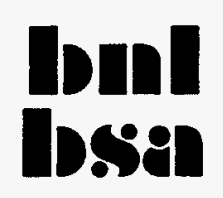

MARCH 25, 1998

\section{RHIC PROJECT}

Brookhaven National Laboratory

Brookhaven Science Associates

Upton, Long Island, New York 11973

Under Contract No. DE-ACO2-98CH10886 with the UNITED STATES DEPARTMENT OF ENERGY 
BNL- 52546

UC-414

\section{THEORY OF TAPERED LASER COOLING}

Hiromi Okamoto and Jie Wei

March 25, 1998

DISTRIBITION OF THIS DOCUMENT IS UNLIMITED fh MASTER

RHIC Project

Brookhaven National Laboratory

Brookhaven Science Associates

Upton, Long Island, New York 11973

Under Contract No. DE-AC02-98CH10886 with the

UNITED STATES DEPARTMENT OF ENERGY 


\section{DISCLAIMER}

This report was prepared as an account of work sponsored by an agency of the United States Government. Neither the United States Government nor any agency thereof, nor any of their employees, not any of their contractors, subcontractors, or their employees, makes any warranty, express or implied, or assumes any legal liability or responsibility for the accuracy, completeness, or usefulness of any information, apparatus, product, or process disclosed, or represents that its use would not infringe privately owned rights. Reference herein to any specific commercial product, process, or service by trade name, trademark, manufacturer, or otherwise, does not necessarily constitute or imply its endorsement, recommendation, or favoring by the United States Government or any agency, contractor, or subcontractor thereof. The views and opinions of authors expressed herein do not necessarily state or reflect those of the United States Government or any agency, contractor or subcontractor thereof.

Printed in the United States of America

Available from

National Technical Information Service

U.S. Department of Commerce

5285 Port Royal Road

Springfield, VA 22161 


\title{
Theory of tapered laser cooling
}

\author{
Hiromi Okamoto \\ Accelerator Laboratory, Institute for Chemical Research, Kyoto University \\ Gokanoshou, Uji, Kyoto 611, Japan
}

Jie Wei*

Brookhaven National Laboratory, Upton, New York 11973, USA

*Work performed under the auspices of the U.S. Department of Energy.

\begin{abstract}
A theory of tapered laser cooling for fast circulating ion beams in a storage ring is constructed. We describe the fundamentals of this new cooling scheme, emphasizing that it might be the most promising way to beam crystallization. The cooling rates are analytically evaluated to study the ideal operating condition. We discuss the physical implication of the tapering factor of cooling laser, and show how to determine its optimum value. Molecular dynamics method is employed to demonstrate the validity of the present theory.
\end{abstract}

\section{INTRODUCTION}

The phase transition of ion beams in a storage ring has attracted a great interest since a sudden jump of the Schottky signal was detected in the NAP-M ring [1]. Due to the recent progress of molecular dynamics (MD) approach, it has been confirmed that beam crystallization is actually possible if one can provide a sufficiently strong 3D cooling force [2]. In the NAP-M case, electron cooling was employed to reduce the beam temperature to $50 \sim 100 \mathrm{~K}$ in the transverse degrees of freedom and $1 \mathrm{~K}$ longitudinally. MD simulations based on the NAP-M condition have demonstrated that the beam behaved like a 1D chain of "disks" formed by transversely oscillating particles [3]. But, in order to reach a ground state, it might be necessary to develop an even better cooling scheme.

Laser cooling [4], whose limiting temperature is of the order of $\mu \mathrm{K}$, could give us the best chance to attain crystalline beams. In fact, recent extensive experimental effort has made it feasible to achieve longitudinal beam temperature in the $\mathrm{mK}$ range $[5,6]$. However, we have so far been 
annoyed by the large imbalance between the transverse and longitudinal temperature as the laser cooling of circulating stored beams operates only one-dimensionally. Although the sympathetic cooling naturally induced by Coulomb interactions has been reported [7], the beam temperatures in the transverse directions are still too high for our final goal.

To overcome this difficulty, several methods applying artificial dynamical coupling have been proposed. The simplest 3D laser cooling scheme is to use momentum dispersion at a regular if cavity installed on a ring [8]. It has been shown both theoretically and numerically that one can enhance the transverse cooling rates up to the same level as the longitudinal rate. Introduction of a coupling cavity operating in $\mathrm{TM}_{210}$ mode is an alternate, mathematically equivalent scheme [9]. We can verify that the coupling cavity even more improves the transverse cooling efficiency. According to the latest $\mathrm{MD}$ study [10], equilibrium transverse temperature achievable with these methods appears to be in the region of $0.01 \sim 1 \mathrm{~K}$, far below the currently available range. As a matter of fact, we have already been successful in observing ordering effects in some numerical experiments where only realistic devices and longitudinal cooling are assumed. It is, however, well-known that these schemes are limited to bunched beams unfortunately. Furthermore, to strengthen the coupling, we must stay close enough to a linear synchro-betatron resonance in every lattice period, which generally makes it troublesome to meet the requirement for crystal maintenance [11]. In the above-mentioned MD simulations, the maintenance condition had actually been broken since we considered only a single if cavity (and a single coupling cavity) on a ring. Consequently, the ordered structures were quickly melted away once the cooling force was removed.

A promising new 3D cooling scheme, tapered cooling, has been explored recently [12]. In this scheme, a properly tapered laser is applied to ion beams. Mathematically, the tapered cooling can be modeled as

$$
\Delta\left(\frac{\delta p}{p}\right)=-f_{s}\left[\left(\frac{\delta p}{p}\right)-\gamma_{x s} \frac{x}{\rho_{m}}\right]
$$

where $\delta p / p$ and $x$ are, respectively, the momentum deviation and horizontal displacement in the laboratory frame, $\rho_{m}$ is the average radius of curvature in the bending section of the storage ring, $\gamma=1 / \sqrt{1-\beta^{2}}$ is the Lorentz factor, $f_{s}$ is a positive constant corresponding to the cooling strength, $\Delta(\delta p / p)$ stands for the change in $\delta p / p$ before and after the laser cooling section, and we call $C_{x s}$ tapering factor [13]. To realize the cooling effect given in Eq. (1), we need to provide a special light whose photon frequency has a linear dependence on the horizontal coordinate. Such a laser could be obtained, for example, by means of a prism. Needless to say, the cooling force can readily be 
extended to the vertical direction simply by setting a skew quadrupole magnet on a ring. As demonstrated below, the tapered cooling scheme enables one to reach all kinds of crystalline structure if the ring lattice satisfies the necessary conditions [11].

In this paper, we give a theoretical description of the physics of tapered cooling. First of all, we present a Hamiltonian formalism for the study of crystalline beams in Sec. II. In Sec. III, the cooling rates are evaluated under the linear approximation, and the stability limit of a laser-cooled beam is discussed. We then show, in Sec. $\mathrm{N}$, how to optimize the tapering factor to crystallize fast circulating beams. Finally, in Sec. V, the theoretical predictions are compared with $\mathrm{MD}$ simulation results.

\section{HAMILTONIAN FORMALISM FOR CRYSTALLINE BEAMS}

Before proceeding to the details of the tapered cooling scheme, let us first construct a Hamiltonian formalism relevant to crystalline-beam study. Although a general theory for spacecharge-dominated beams is available in Ref. [14], we briefly outline, for the sake of completeness, an equivalent formalism under some simplifying assumptions.

The starting point is the variational principle

$$
\delta \int L_{t} d t=0
$$

where $L_{t}$ is the relativistic Lagrangian describing the motion of a charge-particle beam. For later convenience, we change the independent variable from the time $t$ to the reference path length $s$, modifying Eq. (2) to $\delta \int L_{s} d s=0$ in terms of the new Lagrangian

$$
L_{s}=-m c \sqrt{\left(c t^{\prime}\right)^{2}-\mathbf{u}^{\prime} \cdot \mathbf{u}^{\prime}}+q\left(\mathbf{A} \cdot \mathbf{u}^{\prime}-\phi t^{\prime}\right)
$$

where $q$ and $m$ are, respectively, the charge state and rest mass of stored ion, $c$ is the speed of light, $\mathbf{u}$ is the coordinate vector in real space, $\phi$ is the scalar potential of space charges satisfying the Poisson's equation, $\mathrm{A}=\left(A_{x}, A_{y}, A_{s}\right)$ includes the potential of the space-charge field as well as that of external magnetic fields, and the prime stands for differentiation with respect to $s$. In the following analysis, we do not consider rf cavities for simplicity since tapered laser cooling works not only for bunched beams but also for coasting beams. Then, we may approximately put $A_{x}=0=A_{y}$, neglecting the longitudinal component of space-charge force. The Hamiltonian can now be derived, from $L_{s}$, as 


$$
H=-\left(1+\frac{x}{\rho}\right) \sqrt{\left(\frac{p_{t}+q \phi}{c}\right)^{2}-m^{2} c^{2}-p_{x}^{2}-p_{y}^{2}}-q\left(1+\frac{x}{\rho}\right) A_{s},
$$

where $\rho$ is the curvature of the reference orbit, and the longitudinal canonical momentum conjugate to $t$ has been introduced as $p_{t}=\partial L_{s} / \partial t^{\prime}$. Expanding the square root and keeping the low order terms, we obtain

$$
H=-\left(1+\frac{x}{\rho}\right)\left(q A_{s}+p-\frac{p_{x}^{2}+p_{y}^{2}}{2 p}\right)
$$

where $p$ is the total kinetic momentum given by $p=m \beta \gamma c=\sqrt{\left(p_{t}+q \phi\right)^{2} / c^{2}-m^{2} c^{2}}$. Taking only bending and quadrupole magnets into account [15], Eq. (5) becomes

$$
H=-p_{0}\left(1+\frac{x}{\rho}\right) \frac{\Delta E}{\beta_{0}^{2} E_{0}}+\frac{p_{0}}{2 \gamma_{0}^{2}}\left(\frac{\Delta E}{\beta_{0}^{2} E_{0}}\right)^{2}+\frac{p_{x}^{2}+p_{y}^{2}}{2 p_{0}}+\frac{p_{0}}{2}\left(K_{x} x^{2}+K_{y} y^{2}\right)+\frac{q}{\beta_{0} c \gamma_{0}^{2}} \phi
$$

where the quantities with the subscript " 0 " express the values of the reference particle, $K_{x}$ and $K_{y}$ are the horizontal and vertical focusing functions respectively, and $\Delta E=E-E_{0}$ is the total energy difference from the design value $E_{0}$. Using the Suzuki's generating function [16] and then adopting the relative time $\tilde{t}=t-s / \beta_{0} c$ as the longitudinal coordinate, $H$ is transformed to

$$
\begin{aligned}
\tilde{H}= & \frac{1}{2}\left(\tilde{p}_{x}^{2}+K_{x} \tilde{x}^{2}\right)+\frac{1}{2}\left(\tilde{p}_{y}^{2}+K_{y} \tilde{y}^{2}\right)+\frac{1}{2}\left[\frac{1}{\gamma_{0}^{2}}+D_{x}\left(\frac{q}{p_{0} \beta_{0} c \gamma_{0}^{2}} F_{s c}-\frac{1}{\rho}\right)\right]\left(\frac{\Delta \tilde{E}}{\beta_{0} c}\right)^{2} \\
& +\frac{q}{p_{0} \beta_{0} c \gamma_{0}^{2}}\left(\tilde{\phi}-F_{s c} \tilde{x} \frac{\Delta \tilde{E}}{\beta_{0} c}\right)
\end{aligned}
$$

where the new canonical variables of the system are $\left(\tilde{x}, \tilde{p}_{x}, \tilde{y}, \tilde{p}_{y}, \tilde{t}, \Delta \tilde{E}\right)$, the function $\tilde{\phi}$ is related to the original Coulomb potential as $\tilde{\phi}=\phi\left(x=\tilde{x}-D_{x} \Delta \tilde{E} / \beta_{0} c, y=\tilde{y} ; s\right)$, and $F_{s c}$ is the $s$-dependent periodic function associated with the space-charge detuning to the horizontal dispersion function $D_{x}$ [17]; namely,

$$
D_{x}^{\prime \prime}+K_{x} D_{x}=\frac{1}{\rho}+\frac{q}{p_{0} \beta_{0} c \gamma_{0}^{2}} F_{s c}
$$


It is evident from Eq. (7) that the longitudinal canonical momentum is a constant of motion. We therefore write $\Delta \tilde{E} \equiv W=$ const. Moreover, the horizontal betatron equation derived from $\tilde{H}$ suggests that $F_{s c}$ has to be defined by

$$
\frac{W}{\beta_{0} c} F_{s c}=\left(\frac{\partial \tilde{\phi}}{\partial \tilde{x}}\right)_{\tilde{x}=0=\tilde{y}}
$$

such that the space-charge driving terms balance along the closed orbit.

\section{COOLING RATES}

\section{A. Evaluation of the linear cooling rates}

We now try to evaluate the tapered cooling rates in the high-temperature regime. For initial hot beams in the emittance-dominated state, the space-charge potential is negligible. In this case, the one-turn transfer matrix for the canonical variables can be written, by applying the smooth approximation to the Hamiltonian $\tilde{H}$, as

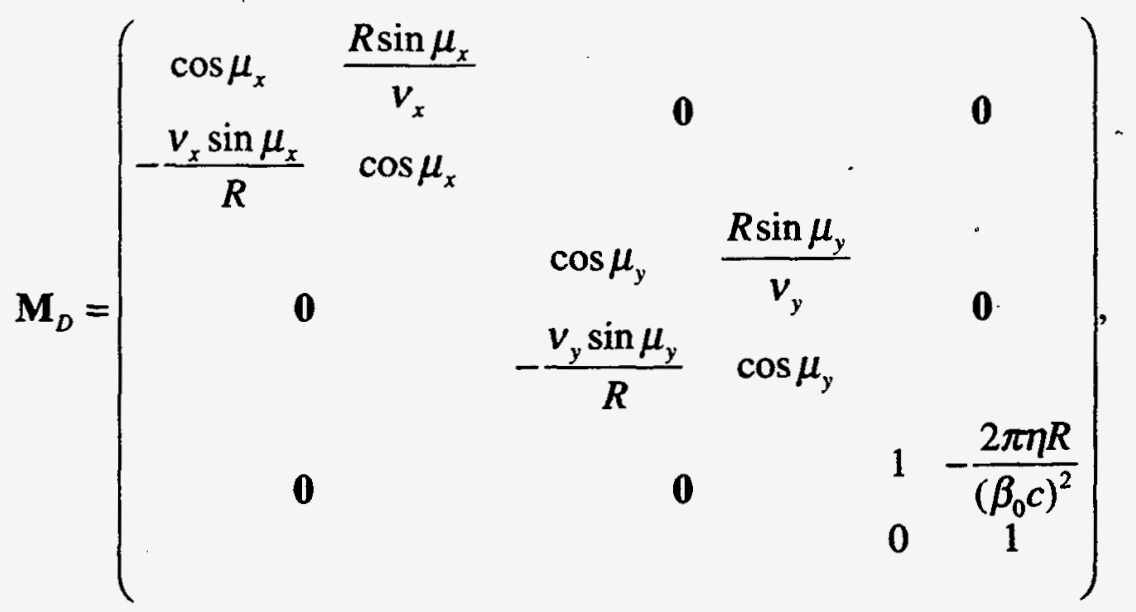

where the horizontal and vertical betatron tunes are denoted by $v_{x}$ and $v_{y}$ respectively, $\eta$ is the phase slip factor, $R$ is the average ring radius, 0 represents the $2 \times 2$ zero matrix, $\mu_{x}=2 \pi v_{x}$, and $\mu_{y}=2 \pi v_{y}$. In addition, from Eq. (1), the effect of the tapered cooling can also be linearized to 


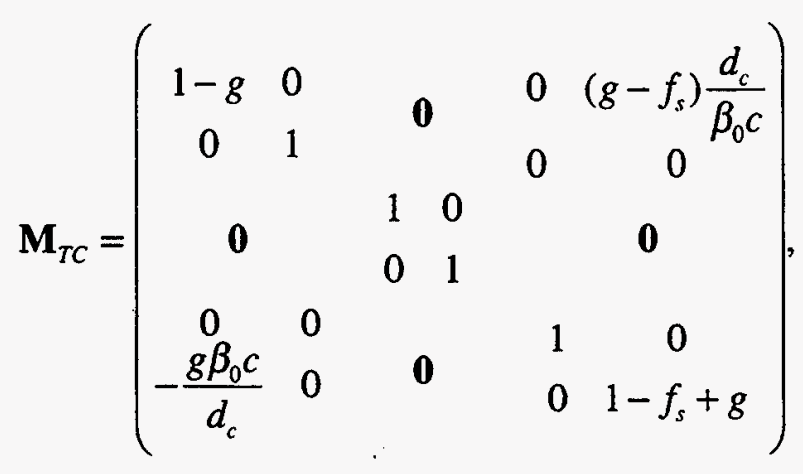

where $d_{c}$ is the magnitude of momentum dispersion in the cooling section, and $g$ is the dimensionless parameter defined by $g \equiv \gamma_{0} f_{s} C_{x s} d_{c} / \rho_{m}$. Specifically, $d_{c}$ must be finite to provide horizontal cooling effect. As mentioned above, a skew quadrupole magnet is needed to combine the vertical motion to other two motions. In the thin lens approximation, the transfer matrix of pure skew field is

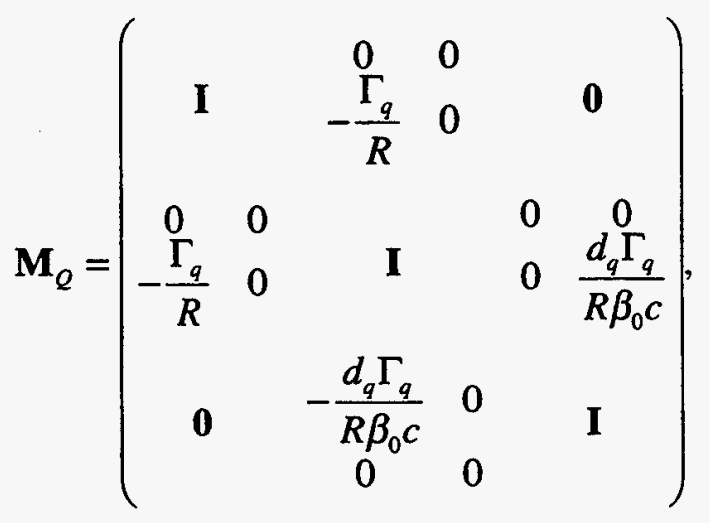

where $d_{q}$ is the dispersion size at the skew magnet, $\mathbf{I}$ denotes the identity matrix, and $\Gamma_{q}$ is the coupling constant. For a quadrupole magnet having the axial length $\ell_{q}, \Gamma_{q}$ is defined by $\frac{\Gamma_{q}}{R}=\frac{\ell_{q}}{B \rho}\left(\frac{\partial B}{\partial v}\right)$ where $B$ is the magnetic field.

In order to know the linear cooling rates, it is only necessary to calculate the eigen values of the matrix $\mathbf{M}=\mathbf{M}_{D} \cdot \mathbf{M}_{Q} \cdot \mathbf{M}_{\tau c}$. Substituting Eqs. (10), (11), and (12) into the characteristic equation $|\mathbf{M}-\lambda \mathbf{I}|=0$, we eventually find the dispersion relation

$$
\begin{array}{r}
\left(\lambda+\frac{1}{\lambda}-2 \cos \mu_{y}\right)\left\{\left(\lambda-1+f_{s}-g\right)\left[\lambda+\frac{1-g}{\lambda}-(2-g) \cos \mu_{x}\right]-g\left(g-f_{s}\right)\left(\frac{1}{\lambda}-\cos \mu_{x}\right)\right\} \\
-\Gamma\left\{\left[1-\left(1-\frac{d_{q}}{d_{c}}\right) g\right] \lambda-1+f_{s}\right\}=0
\end{array}
$$


where $\Gamma \equiv\left(2 \pi \Gamma_{q}\right)^{2} \sin \mu_{x} \sin \mu_{y} / \mu_{x} \mu_{y}$. When writing $\lambda=\exp (i \mu)$, the imaginary part of $\mu$ can directly be related to the cooling rates. A larger $\operatorname{Im}(\mu)$ implies a higher cooling rate while beam becomes unstable if $\operatorname{Im}(\mu)<0$. As an example, we illustrate, in Fig. 1, typical solutions of Eq. (13) under a strong cooling situation. We recognize that one of the three cooling rates turns to negative beyond a certain $C_{x s}$; in other words, too steep tapering causes beam heating. It can be shown that there always exists a threshold value of $C_{x s}$.
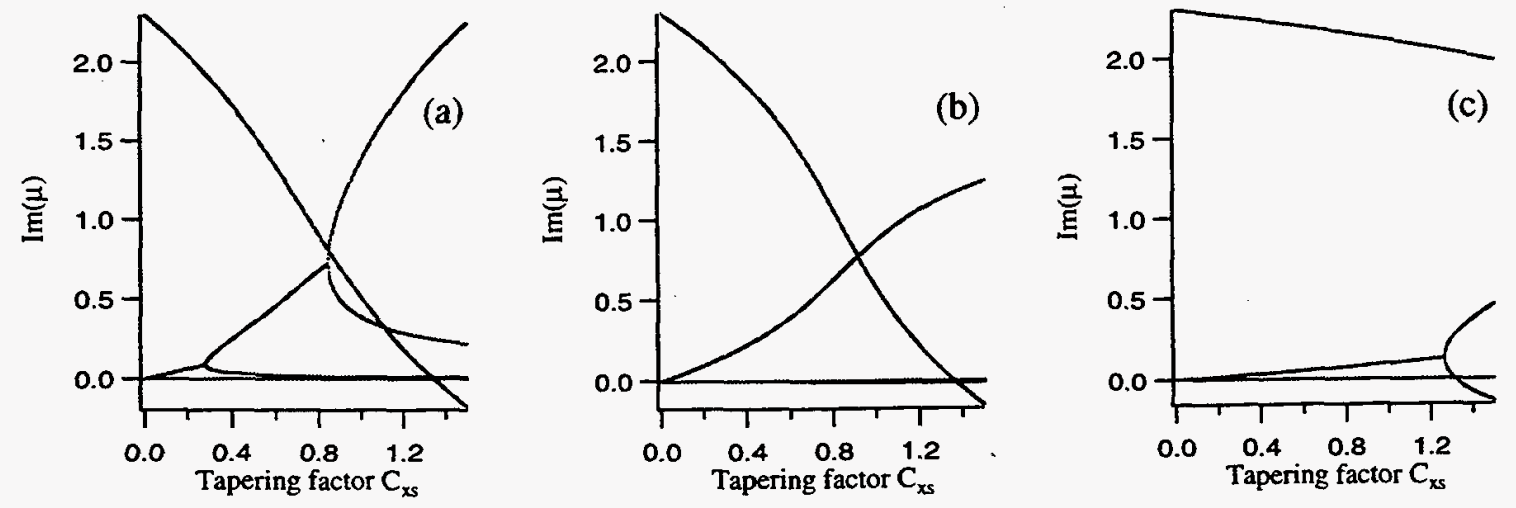

Fig. 1 Cooling rates vs. tapering factor $C_{x s}$ in the case where $f_{s}=0.9$. We have assumed $1 \mathrm{MeV}^{24} \mathrm{Mg}^{+}$beam stored in a storage ring with $R=12.366 \mathrm{~m}$ and $\rho_{m}=4.01 \mathrm{~m}$. Three dfferent sets of betatron tunes have been considered (a). $v_{x}=v_{y}=1.4$, (b). $v_{x}=1.7$ and $v_{y}=2.1$, (c). $v_{x}=2.1$ and $v_{y}=2.2$. Other lattice parameters employed for these pictures are: $d_{c}=d_{q}=3 \mathrm{~m}, \eta=-0.7$, and $\Gamma_{q}=0.247$.

\section{B. Operating point consideration and limiting tapering factor}

It is interesting to see that the cooling rates of all three directions exhibit linear dependence on the tapering factor when $f_{s}$ is not too large. For instance, assuming $f_{s}$ to be 0.1 instead of 0.9 , Fig. 1 is altered to Fig. 2. Of note here is the fact that the vertical cooling rate is almost zero in Figs. 2 (b) and 2 (c). On the other hand, both transverse cooling rates are roughly equal in the case of Fig. 2 (a) where the betatron tunes are on resonance. We have numerically confirmed that this is a general tendency in the case of modest cooling. Thus, in order to have the maximum cooling effect, it may be preferable to set $v_{x} \approx v_{y}$.

Provided that $v_{x} \approx v_{y}$ and $\Gamma \ll 1$, it is possible to solve Eq. (13) in a perturbative way. Assuming $d_{c} \approx d_{q}$ for simplicity, the cooling rates can be obtained as 


$$
\begin{cases}\operatorname{Im}(\mu) \approx G C_{x s} & \text { (for the transverse motions) } \\ \operatorname{Im}(\mu) \approx f_{s}-4 G C_{x s} & \text { (for the longitudinal motion) }\end{cases}
$$

where $G \equiv \gamma_{0} d_{c} f_{s} / 4 \rho_{m}$. As expected, the cooling rates are linearly proportional to $C_{x s}$. Notice further that they are independent of the skew coupling factor $\Gamma_{q}$. These analytic formulas agree well with the exact solutions numerically evaluated from the dispersion relation.

Eqs. (14) also tells us that the stable operating region is given by

$$
C_{x s}<\frac{\rho_{m}}{\gamma_{0} d_{c}}
$$

which is even independent of the cooling strength $f_{s^{\prime}}$. Substituting $\rho_{m}=4.01 \mathrm{~m}, d_{c}=3 \mathrm{~m}$, and $\gamma_{0}=1$ into Eq. (15), the limiting tapering factor is calculated to be 1.34. Obviously, this prediction is in good agreement with the numerical results displayed in both Figs. 1 and 2.
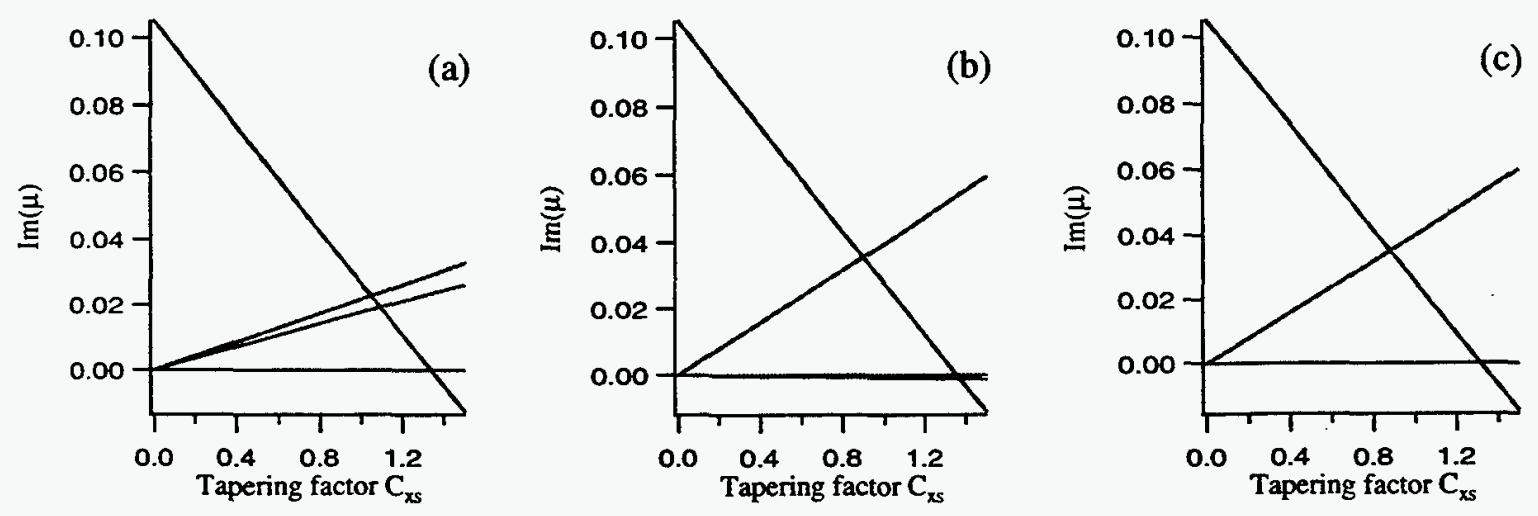

Fig. 2 Cooling rates vs. tapering factor $C_{x s}$ in the case where $f_{s}=0.1$. All parameters except for $f_{s}$ are the same as those employed in Fig. 1. In the case (b) and (c), the vertical cooling rate is close to zero.

\section{OPTIMIZATION OF THE TAPERING FACTOR}

\section{A. Physical meaning of $C_{x}$ s}

According to Eqs. (14), it may be concluded that a preferred operating point corresponds to the intersection of the two cooling-rate lines where $C_{x s}=f_{s} / 5 G$. However, as approaching an ultracold state, we will be forced to carefully incorporate the space-charge force which dominates the beam. 
To determine the optimum cooling condition in consideration of Coulomb interactions, we here think of the physical implication of the tapering factor, going back to the formula in Eq. (1). Once a final equilibrium state has been reached due to the tapered cooling, $\Delta(\delta p / p)$ in Eq. (1) would vanish, leading to $\delta p / p=\gamma C_{x s} x / \rho_{m}$. This condition is approximately equivalent to

$$
\frac{\beta-\beta_{0}}{\beta_{0}}=C_{x s} \frac{x}{\gamma_{0} \rho_{m}}
$$

which implies that an outer particle has a velocity greater than that of an inner particle. This can quite easily be understood if we imagine the nature of crystalline beams. In order for a crystalline beam to maintain its ordered structure, all particles must have the same revolution frequency. Therefore, an outer particle has to run faster than an inner particle. Figure 3 shows the phase-space profile of a typical 3D crystal. We clearly observe the linear dependence of the longitudinal momentum on the horizontal coordinate. This observation also suggests that too strong un-tapered cooling force may destroy multi-dimensional crystalline structures since it tries to equalize the longitudinal velocities of all particles.

It is straightforward to estimate the ground-state value of $C_{x s}$. First of all, note that the revolution time of a particle with the velocity $\beta c$ can be given by

$$
T=\frac{1}{\langle\beta\rangle c} \int_{0}^{2 \pi R} d s \sqrt{\left(\frac{d x}{d s}\right)^{2}+\left(1+\frac{x}{\rho}\right)^{2}}
$$

where $\langle A\rangle$ stands for taking the average of the quantity $A$ over one turn, and we have looked at a particle moving on the horizontal plane. Requiring $T$ to be equal to the design revolution time $2 \pi R / \beta_{0} c$, Eq. (17) together with Eq. (16) yield

$$
\Delta L \approx \frac{2 \pi R}{\gamma_{0} \rho_{m}}\left\langle C_{x s} x\right\rangle
$$

where $\Delta L$ is the path-length difference defined by

$$
\Delta L=\int_{0}^{2 \pi R} d s \sqrt{\left(\frac{d x}{d s}\right)^{2}+\left(1+\frac{x}{\rho}\right)^{2}}-2 \pi R
$$


Recalling that $|x / \rho| \ll 1$ and $|d x / d s| \ll 1$, the integral in Eq. (19) takes a finite value, in the first-order approximation, only within bending regions. Then, assuming that $x \approx\langle x\rangle$ in dipole magnets, Eqs. (18) and (19) result in

$$
\bar{C}_{x s} \equiv \frac{\left\langle C_{x s} x\right\rangle}{\langle x\rangle} \approx \frac{\gamma_{0} \rho_{m}}{R} .
$$

Although Eq. (20) allows us to make a rough estimate of the optimum tapering gradient, it inevitably contains some error because of the $s$-dependence of $C_{x s}$ neglected here. To improve the accuracy of this prediction, we have to multiply Eq. (20) by a correction factor. For example, we may introduce the factor $\zeta_{c}$ as the ratio of the average dispersion to $d_{c}$; namely, $\zeta_{c} \equiv R / \gamma_{T}^{2} d_{c}$ where $\gamma_{T}$ is the transition energy of the ring. This definition of the correction factor makes sense since the orbit of each particle in a ground state is basically determined by momentum dispersion as discussed later.
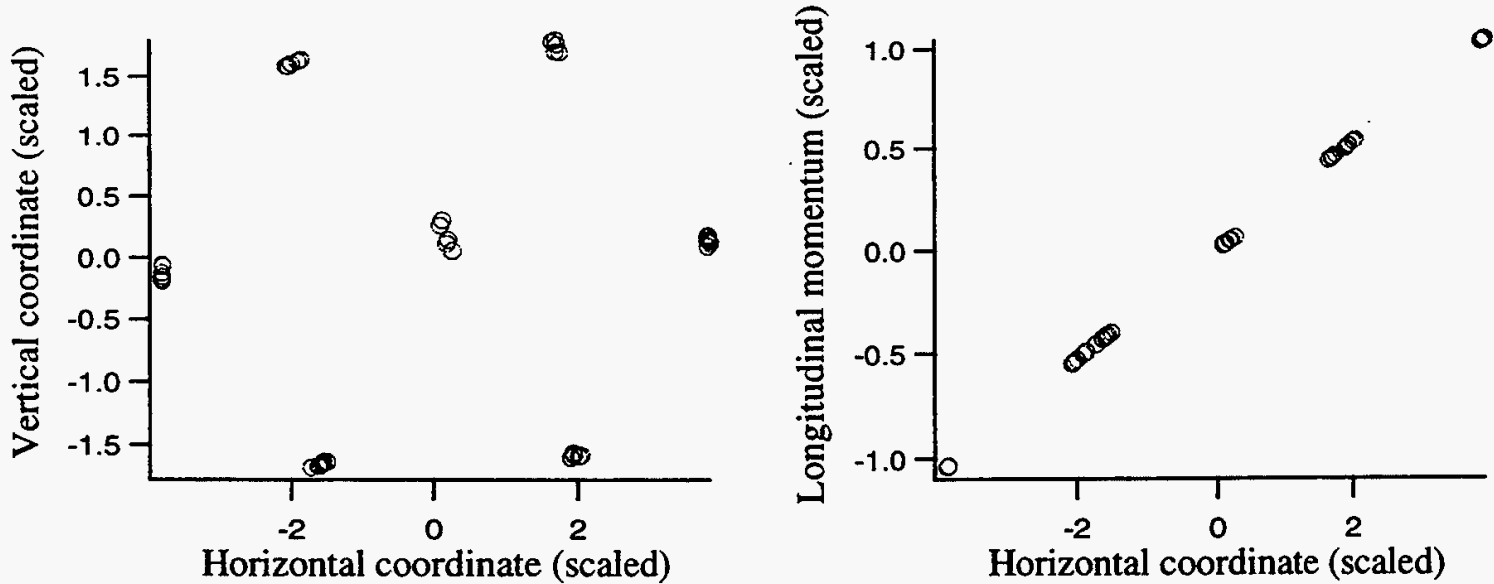

Fig. 3 Real-space and phase-space configurations of a single-shell crystalline beam. The lattice parameters of the storage ring TARN II [18] has been considered in this molecular dynamics simulation. The stored ions are $1 \mathrm{MeV}^{24} \mathrm{Mg}^{+}$, and the betatron tunes have been adjusted to $v_{x}=v_{y}=1.7$.

\section{B. Analytic determination of the optimum $C_{x s}$}

In this section, we step forward to show how to predict the almost exact value of the optimum $C_{x s}$ at an arbitrary position along the design orbit. For this purpose, let us investigate the theoretical basis as to how the revolution frequencies of all stored particles become identical in a 
ground state. Noting that the real-space density of a crystalline beam is roughly uniform, the mean space-charge potential might be dominated by the linear force terms. In other words, we may approximately leave only the quadratic terms in the potential $\phi$. Then, the dynamical system of our interest turns out to be perfectly self-consistent, provided the space-charge potential satisfies

$$
\phi=-\frac{q N}{2 \pi \varepsilon_{0}}\left[\frac{x^{2}}{a(a+b)}+\frac{y^{2}}{b(a+b)}\right]
$$

where $N$ is the number of particles per unit length. Here, $a$ and $b$ denote, respectively, the horizontal and vertical beam size governed by the KV-type envelope equations [19]

$$
a^{\prime \prime}+K_{x} a-\frac{\varepsilon_{x}^{2}}{a^{3}}=\frac{2 K_{s c}}{a+b}, \quad b^{\prime \prime}+K_{y} b-\frac{\varepsilon_{y}^{2}}{b^{3}}=\frac{2 K_{s c}}{b+a}
$$

where $\varepsilon_{x}$ and $\varepsilon_{y}$ are the transverse beam emittances, and $K_{s c} \equiv 2 N r_{p} / \beta_{0}^{2} \gamma_{0}^{3}$ with $r_{p}$ being the classical radius of the particle. The Hamiltonian in $\mathrm{Eq}$. (7) is then reduced to the simple form

$$
\tilde{H}=\frac{1}{2}\left(\frac{1}{\gamma_{0}^{2}}-\frac{D_{x}}{\rho}\right) \frac{W^{2}}{\left(\beta_{0} c\right)^{2}}+\frac{\tilde{p}_{x}^{2}+\tilde{p}_{y}^{2}}{2}+\frac{1}{2}\left[K_{x}-\frac{2 K_{s c}}{a(a+b)}\right] \tilde{x}^{2}+\frac{1}{2}\left[K_{y}-\frac{2 K_{s c}}{b(a+b)}\right] \tilde{y}^{2},
$$

indicating that the definition of the momentum compaction factor $\alpha_{s c}$ does not change; namely, we can define it to be $\alpha_{s c}=(2 \pi R)^{-1} \oint d s\left(D_{x} / \rho\right)$. Furthermore, we have, from Eqs. (8), (9), and (21),

$$
D_{x}^{\prime \prime}+\left[K_{x}-\frac{2 K_{s c}}{a(a+b)}\right] D_{x}=\frac{1}{\rho}
$$

Since the momentum dispersion can strongly be detuned by the space-charge term in Eq. (24), $\alpha_{s c}$ suffers a considerable change as approaching a ground state. In this way, the isochronous condition $\alpha_{s c}=1 / \gamma_{0}^{2}$ is naturally accomplished in crystalline beams such that all particles have an identical revolution period.

Apparently, betatron motion has been completely suppressed in ground states, which means that the coordinate $\tilde{x}$ is very close to zero. Recalling that $x=\tilde{x}-D_{x} W / \beta_{0} c$, the transverse trajectory mainly originates from the dispersion function, i.e. $x \approx-D_{x} W / \beta_{0} c$. Substitution of this formula into 
Eq. (16) leads to $\left(\beta-\beta_{0}\right) c \approx-W C_{x s} D_{x} / \gamma_{0} \rho_{m}$. This equation can be rewritten as

$$
C_{x s} \approx \frac{\rho_{m}}{\gamma_{0} D_{x}}
$$

Thus, in order to know the optimum tapering factor along the reference orbit, we only need to solve Eq. (24) together with Eqs. (22) for the dispersion function $D_{x}$ and, then, substitute the result into Eq. (25).

We readily notice, from the isochronous condition, that the average dispersion in a ground state is roughly equal to $R / \gamma_{0}^{2}$. Therefore, by taking the average of Eq. (25) over one turn, we obtain $\left\langle C_{x s}\right\rangle \approx \gamma_{0} \rho_{m} / R$, consistent with Eq. (20). Equation (25) also gives us a simple interpretation of the limiting tapering factor discussed in Sec. III B. For an initial hot beam, the dispersion in the cooling section is $d_{c}$. On the other hand, the tapered cooling force pushes off-momentum particles onto particular closed orbits determined by the space-charge-detuned dispersion function $D_{x}$ in Eq. (24). To prevent an input beam from average momentum increase after the interaction with a tapered laser, the ground-state value of $D_{x}$ must be greater than $d_{c}$; i.e., $D_{x}>d_{c}$ in Eq. (25). We thus conclude again $C_{x s}<\rho_{m} / \gamma_{0} d_{c}$ for beam stability. Note that this argument as well as Eq. (25) does not depend on the cooling strength $f_{s}$. Hence, the condition in Eq. (15) should be satisfied in any situations. As a matter of fact, Fig. 1 has already pointed out that Eq. (15) holds even for a large $f_{s}$.

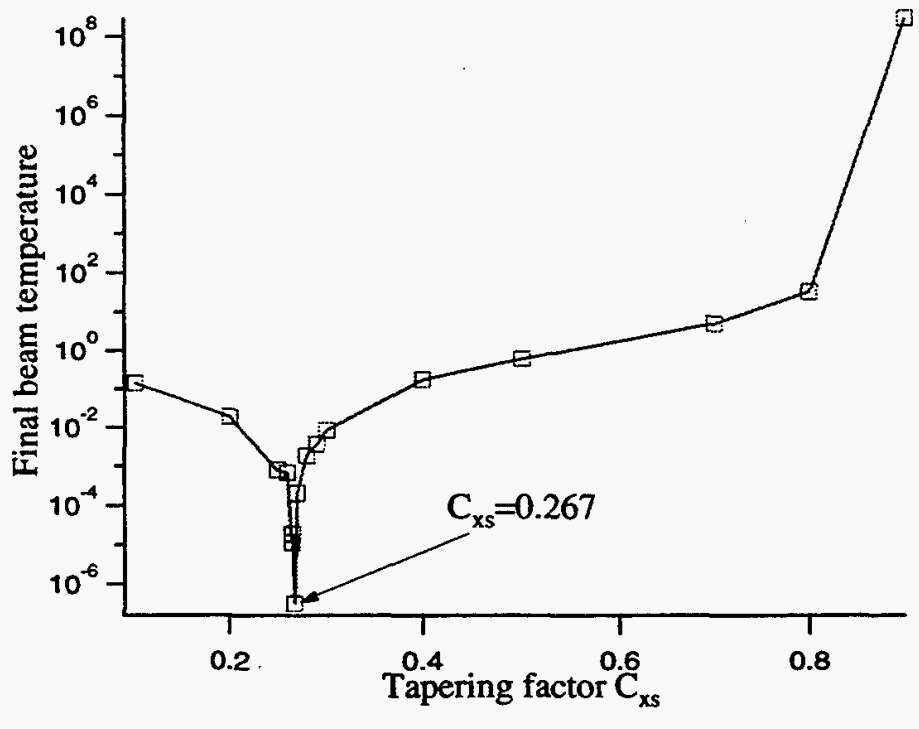

Fig. 4 Typical dependence of equilibrium beam temperature on the tapering factor. The result was obtained with the MD code [3], assuming $1 \mathrm{MeV}$ ${ }^{24} \mathrm{Mg}^{+}$beam stored in TARN II. The total number of the stored ions is $N_{\text {tot }}=3.38 \times 10^{6}$. Here, $v_{x}=1.68$, $v_{y}=1.85$, and we have installed two skew quadupoles in each lattice period, whose skew strength factor is $\Gamma_{q}=0.124$. The optimum tapering factor found in this simulation is 0.267 at which the beam has been completely crystallized. For the definition of the beam temperature, see, e.g., Ref. [3]. 


\section{MOLECULAR DYNAMICS RESULTS}

At present, there exist only two storage rings in the world where laser cooling system has been installed; namely, the TSR ring [5] at MPI, Heidelberg, and the ASTRID ring [6] at Aarhus University, Denmark. However, these storage rings do not satisfy the necessary condition of crystal maintenance although with minor modifications they would. Thus, in order to give specific numerical results, we here choose the TARN II parameters as an example [18]. The cooler ring "TARN II", whose circumference is about $77.7 \mathrm{~m}$, has the superperiodicity of 6 and contains four bending magnets in each lattice period. The orbit curvature in the bending regions is $4.01 \mathrm{~m}$. Since the operating betatron tune is usually set at around two or even less, this ring is crystal friendly.

A typical $C_{x s}$-dependence of final beam temperature in TARN II is displayed in Fig. 4. We observe a sharp drop of temperature at $C_{x s}=0.267$ where the beam has been fully crystallized. It is also recognized that the beam has heated up when $C_{x s}$ exceeds around 0.9 . Noting that $\rho_{m}=4.01 \mathrm{~m}$, $\gamma_{0}=1.000044$, and $d_{c}=4.09 \mathrm{~m}$, Eq. (15) predicts the stability border of 0.98 which is in good agreement with this $\mathrm{MD}$ result. Concerning the optimum size of the tapering gradient, Eq. (20) multiplied by $\zeta_{c}$ yields $\zeta_{c} \bar{C}_{x s} \approx 0.280$. We again find a nice agreement with the exact value 0.267 while $\bar{C}_{x s}$ is only a rough estimate based on the first-order approximation. Without the correction factor, we have $\bar{C}_{x s} \approx 0.324$.

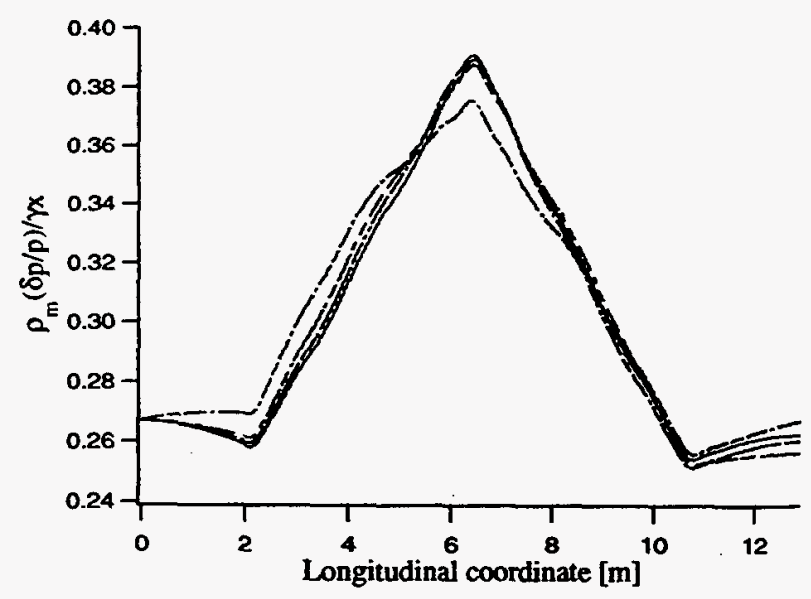

Fig. 5 Variation of the function $\rho_{m}(\delta p / p) / \not x$ along the reference orbit. $\rho_{m}(\delta p / p) / \gamma x$ of ten particles, calculated from a MD result, have been plotted We have taken into account the same TARN II parameters as used in Fig. 4, setting $C_{x}=0.267$.

Figure 5, obtained from a MD simulation, illustrates the longitudinal variation of $\rho_{m}(\delta p / p) / \gamma x$ of ten arbitrarily picked particles within the last lattice period. Apart from small thermal noises, all particles have an almost identical $\rho_{m}(\delta p / p) / \not x$ just corresponding to the optimum tapering factor. In this simulation, the cooling section is located at both ends of the picture, consistent 
with the fact that $C_{x s} \approx 0.267$ at optimum. The task now is to examine whether the theory proposed can reproduce the curve in Fig. 5. To do this, let us rewrite Eqs. (22), for convenience, as

$$
\tilde{a}^{\prime \prime}+K_{x} \tilde{a}-\frac{\tilde{\varepsilon}_{x}^{2}}{\tilde{a}^{3}}=\frac{2}{\tilde{a}+\tilde{b}}, \quad \tilde{b}^{\prime \prime}+K_{y} \tilde{b}-\frac{\tilde{\varepsilon}_{y}^{2}}{\tilde{b}^{3}}=\frac{2}{\tilde{b}+\tilde{a}}
$$

where $\tilde{a}=a / \sqrt{K_{s c}}, \tilde{b}=b / \sqrt{K_{s c}}, \tilde{\varepsilon}_{x}=\varepsilon_{x} / K_{s c}$ and $\tilde{\varepsilon}_{y}=\varepsilon_{y} / K_{s c}$. Similarly, the parameter $K_{s c}$ is eliminated from Eq. (24) as

$$
D_{x}^{\prime \prime}+\left[K_{x}-\frac{2}{\tilde{a}(\tilde{a}+\tilde{b})}\right] D_{x}=\frac{1}{\rho} .
$$

For the TARN II example considered in this section, we may set $\tilde{\varepsilon}_{x} \approx \tilde{\varepsilon}_{y}(\equiv \tilde{\varepsilon})$ since the betatron tunes are close to each other. To choose a proper value of $\tilde{\varepsilon}$, we need to know the phase-space density of crystalline beam [20]. It is, of course, possible but we here try an even easier way to evaluate the density parameter; namely, the isochronous condition is employed to search for the correct magnitude of $\tilde{\varepsilon}$. After some straightforward numerical procedure, we conclude that $\tilde{\varepsilon}$ must be about 5.4 in the present case. Then, calculating the periodic solutions of Eqs. (26) and substituting the result in Eq. (27), we obtain the dispersion function as plotted in Fig. 6 (a). It is now obvious that the spacecharge-detuning effect on momentum dispersion can be quite large in a high-density beam. The func-
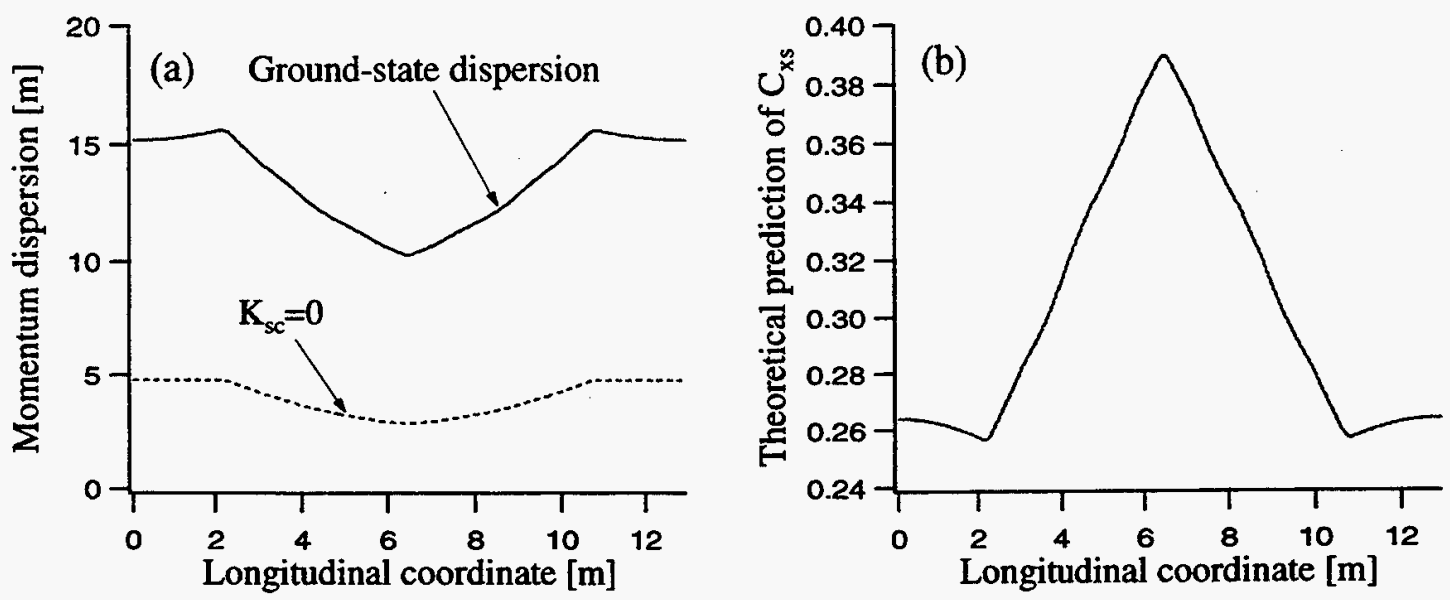

Fig. 6 Theoretical predictions. (a). The solid line corresponds to the momentum dispersion in a ground state while the dotted line to that in the absence of space charge, i.e. the case of $K_{s c}=0$. (b). The optimum tapering factor along the reference orbit, predicted by Eq. (25). 
tion $D_{x}$ shown in Fig. 6 (a) is further substituted in Eq. (25) to generate Fig. 6 (b) where we observe an excellent agreement with the MD results in Fig. 5. This inversely indicates that the isochronous condition is certainly realized in ground states, and also that ground-state dispersion can uniquely be determined once the lattice structure of a storage ring is fixed.

It is practically meaningful to check out how much error in $C_{x s}$ will be acceptable to achieve beam crystallization. The definition of the threshold temperature is, however, a sort of arbitrary because no sharp phase transition has so far been encountered in coasting beams [21]. Therefore, we simply assume here that a normalized temperature below 0.05 is necessary to attain a crystalline beam. This assumption should be reasonable as strong ordering between particles actually occurs around this temperature according to past MD study. Furthermore, it has also been confirmed that the survival time of a crystalline beam is sufficiently long below this threshold. Figure 7 shows the upper and lower limit of the acceptable tapering factor determined in this way. It is evident that the acceptable range becomes particularly large for $1 \mathrm{D}$ crystals. We have further verified, based on systematic MD simulations, that this range is quite insensitive to the value of $f_{s}$.

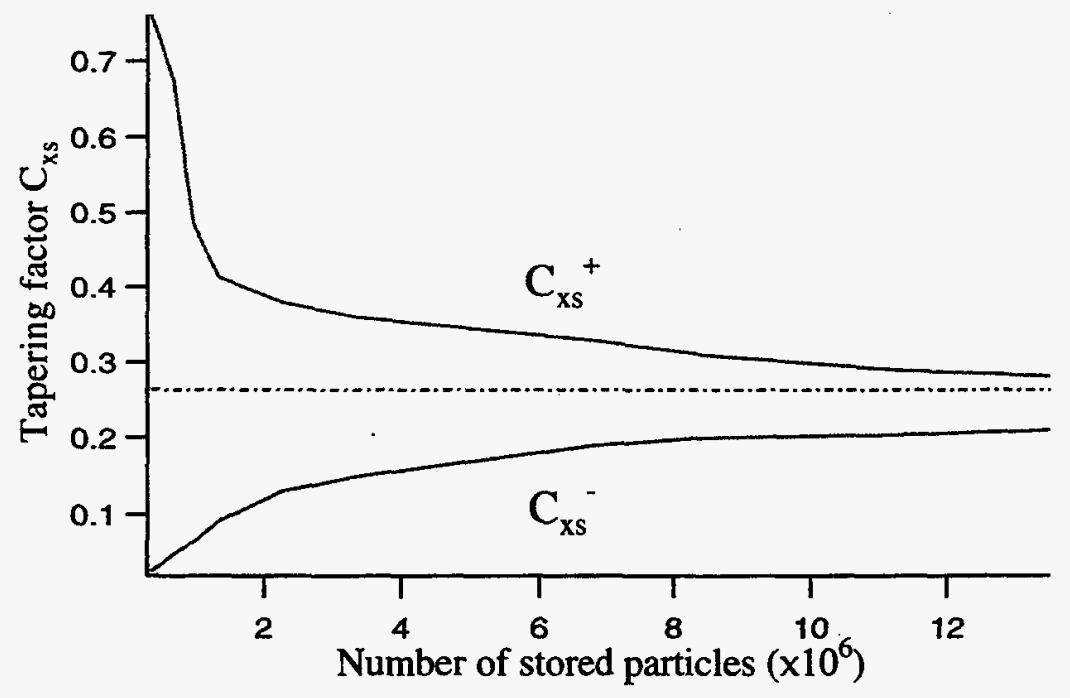

Fig. 7 Acceptable range of tapering factor. $C_{x s}{ }^{+}$and $C_{x}{ }^{-}$represent, respectively, the upper and lower limit of $C_{x s}$ required for beam crystallization. The dotdash line indicates the ideal value predicted by the theory. Note that the transition from $1 \mathrm{D}$ to $3 \mathrm{D}$ crystalline structure takes place when the number of stored particles is around $1.2 \times 10^{5}$ in the present example.

\section{CONCLUDING REMARKS}

Finally, it is worthy to make a remark upon the self-consistency of our theory. Of essential importance is that, in deriving Eqs. (21) and (22), we have assumed the Vlasov equation $d f / d s=0$ where $f$ is phase-space distribution function. On the other hand, it is commonly believed that intrabeam scattering naturally plays an increasing role as the beam is compressed during the cooling process. If we follow the latter understanding, the collision term should be added to the Vlasov 
equation for a correct treatment of cold beams. Nevertheless, why does the Vlasov equation suffice for describing the behavior of crystalline beams ? An answer can be found in Fig. 8 where the heating rates of bunched and coasting beams at different line density have been plotted. Figure 8 shows that the heating effect due to random collisions actually grows rapidly as an initial hot beam is cooled down to a low-temperature state. However, once the beam is cooled beyond the peak of the heatingrate curve, the collisional effect starts to be monotonically weakened and has eventually disappeared when arriving at a ground state. This observation suggests that, for crystalline beams, Coulomb interactions between individual particles can be smoothed out in terms of collective average. This is why Eq. (24) together with Eq. (22) allow an accurate prediction to the optimum tapering factor. Furthermore, we can easily prove that the condition of crystal maintenance is a natural consequence from Eq. (22) [14].

To conclude, we have given a satisfactory theoretical description of tapered laser cooling. Considering the nature of ground states, it has been demonstrated that this new cooling scheme provides a promising possibility of crystallizing fast circulating beams in a storage ring. The linear cooling rates have been evaluated from a characteristic equation. A Hamiltonian treatment has also been established to understand some important properties of crystalline beams. In particular, we have shown how the revolution frequencies of all particles in a ground state converge on the design value. Space-charge-detuned dispersion function and the isochronous condition have been employed to predict the optimum value of the tapering factor at an arbitrary location along the design orbit. It has been found that the theoretical predictions given here are in excellent agreement with results from molecular dynamics calculations.

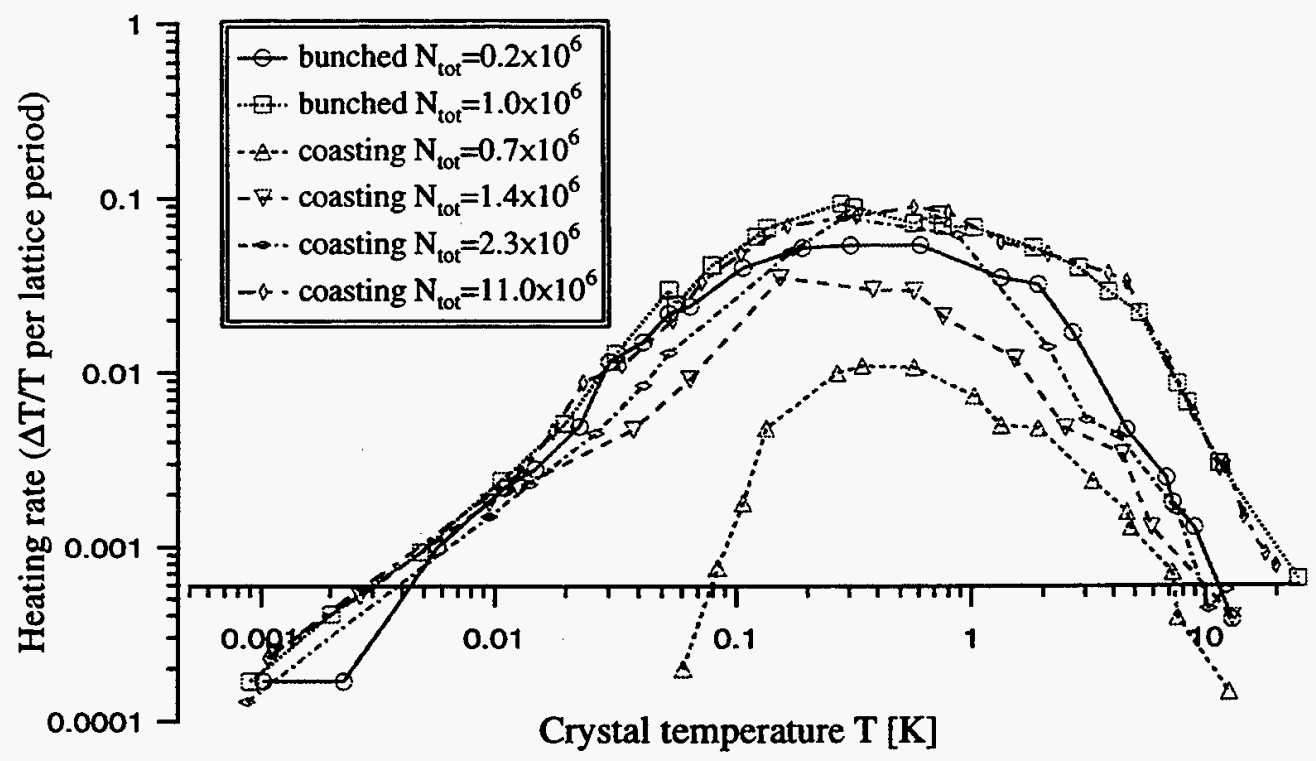

Fig. 8 Heating rate curves for bunched and coasting beams at different line density. 


\section{ACKNOWLEDGMENT}

The authors would like to thank Dr. X.-P. Li for originally developing the MD simulation program. They would also like to express their sincere gratitude to Dr. A. M. Sessler for his stimulating suggestions in the course of this work. One of the authors (HO) is indebted to Prof. S. Y. Lee for valuable discussions on space-charge-dominated beam dynamics.

\section{REFERENCES}

[1]. V. V. Parkhomchuk and N. S. Dikansky, Sov. J. of Tech. Phys., bf 50, 1411 (1980); E. E. Dement'ev, N. S. Dikansky, A. S. Medvenko, V. V. Parkhomchuk, and D. V. Pestrikov, Zh. Tekh. Fiz. 50, 1717 (1980).

[2]. A. Rahman and J. P. Schiffer, Phys. Rev. Lett. 57, 1133 (1986); R. W. Hasse and J. P. Schiffer, Ann. Phys. 203, 419 (1990); J. P. Schiffer, Phys. Rev. Lett. 70, 818 (1993); J. Wei, X-P. Li, and A. M. Sessler, Brookhaven National Laboratory Report BNL-52381, Upton, New York (1993); J. Wei, X.-P. Li, and A. M. Sessler, Phys. Rev. Lett. 73, 3089 (1994).

[3]. J. Wei, A. Draeseke, A. M. Sessler, and X.-P. Li, Crystalline Beams and Related Issues, published by World Scientific Publishing Co. Pte. Ltd., ISBN 981-02-2785-X, 229 (1996).

[4]. D. J. Wineland and H. Dehmelt, Bull. Am. Phys. Soc. 20, 637 (1975); T. Hänsch and A. Schawlow, Opt. Commun. 13, 68 (1975).

[5]. S. Schröder, R. Klein, N. Boos, M. Gerhard, R. Grieser, G. Huber, A. Karafillidis, M. Kieg, N. Schmidt, T. Kühl, R. Neumann, V. Balykin, M. Grieser, D. Habs, E. Jaeschke, D. Krämer, M. Kristensen, M. Music, W. Petrich, D. Schwalm, P. Sigray, M. Steck, B. Wanner, and A. Wolf, Phys. Rev. Lett. 64, 2901 (1990).

[6]. J. S. Hangst, M. Kristensen, J. S. Nielsen, O. Poulsen, J. P. Schiffer, and P. Shi, Phys. Rev. Lett. 67, 1238 (1991).

[7]. H- J. Miesner, R. Grimm, M. Grieser, D. Habs, D. Schwalm, B. Wanner, and A. Wolf, Phys. Rev. Lett. 7 7, 623 (1996).

[8]. H. Okamoto, Phys. Rev. E50, 4982 (1994).

[9]. H. Okamoto, A. M. Sessler, and D. Möhl, Phys. Rev. Lett. 72,3977 (1994).

[10]. T. Kihara, H. Okamoto, Y. Iwashita, J. Wei, K. Oide, A. M. Sessler, to be published.

[11]. To form and maintain a crystalline beam, the storage-ring lattice must satisfy the following two conditions: (a). the beam energy is smaller than the transition energy of the ring and (b). the lattice superperiodicity divided by $2 \sqrt{2}$ is greater than both betatron tunes. See, e.g., J. Wei, X.-P. Li, and A. M. Sessler, Advanced Accelerator Concepts, AIP Conf. Proc. 335, (Fontana WI, 1994), 
edited by P. Schoessow.

[12]. J. Wei, H. Okamoto, and A. M. Sessler, Phys. Rev. Lett. 80, 2606 (1998).

[13]. This equation is equivalent to Eq. (4) in Ref. [12].

[14]. S. Y. Lee and H. Okamoto, submitted to Phys. Rev. Lett.

[15]. In the tapered cooling scheme, the installation of skew quadrupole magnets is usually required to couple the vertical motion to the horizontal motion. However, as we will see later, the general properties of tapered cooling can be well described by the present Hamiltonian unless the skew field is too strong.

[16]. T. Suzuki, Part. Accel. 12, 237 (1982).

[17]. In general, the space-charge potential contains the linear cross term which gives rise to vertical dispersive effects. In such a case, it is convenient to define the vertical dispersion function by introducing the generalized Suzuki's function (See Ref. [14]). For crystalline beams, however, the vertical dispersion is mostly negligible due to the symmetry of the configuration.

[18]. T. Katayama et al., "Present Status of Cooler Ring TARN II", Proc. of European Particle Accelerator Conf., (Nice, 1990) 577.

[19]. I. M. Kapchinskij and V. V. Vladimirskij, Proc. of the Int. Conf. on High Energy Accelerators, (CERN, Geneva, 1959) 274.

[20]. For a larger beam density, $\tilde{\varepsilon}$ becomes smaller.

[21]. The situation might be quite different for bunched beams since they are substantially threedimensional. 


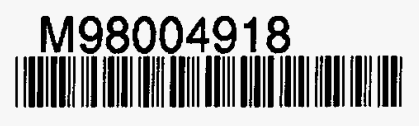

Report Number (14) BNL--52546

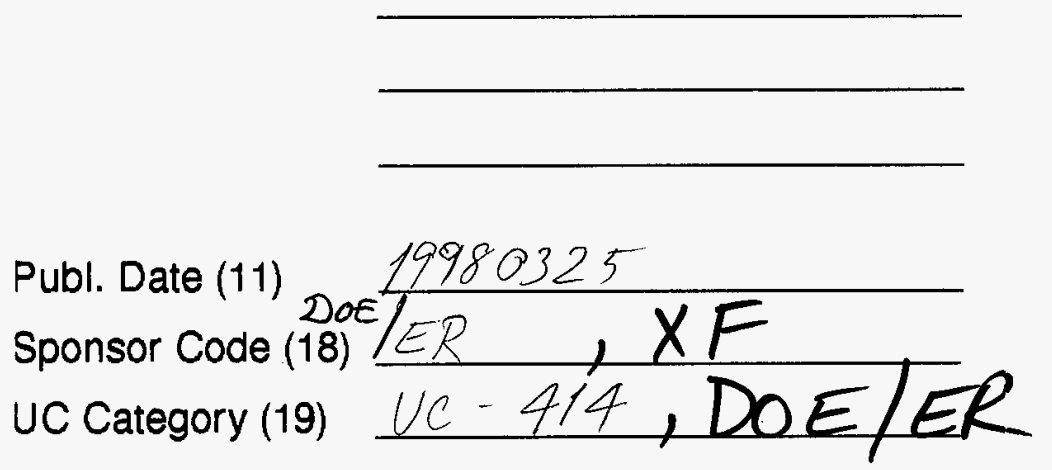

19980622020 